Physics

Physics Research Publications

\title{
Friedel oscillations in a Fermi liquid
}

\author{
G. E. Simion \\ G. F. Giuliani
}




\title{
Friedel oscillations in a Fermi liquid
}

\author{
George E. Simion and Gabriele F. Giuliani \\ Physics Department, Purdue University, West Lafayette Indiana 47907, USA
}

(Received 10 February 2005; published 19 July 2005)

\begin{abstract}
The problem of the Friedel oscillations in two- and three-dimensional electron Fermi liquids is examined by means of the many-body local fields theory as aided by the most recent results of accurate numerical studies based on the quantum Monte Carlo method. Within linear response, an exact answer is obtained for the amplitude of the electron density distortion due to both short- and long-ranged impurity potentials. It is discussed how a measurement of the local environment of an impurity embedded in an otherwise homogeneous electron liquid can be used to characterize the systems as a Fermi liquid as well as to extract the magnitude of the static many-body corrections beyond the random phase approximation for wave vector equal to $2 k_{F}$.
\end{abstract}

DOI: 10.1103/PhysRevB.72.045127

\section{INTRODUCTION}

Recent advances in both experimental techniques and theoretical methods have rekindled considerable interest in the problem of the Friedel oscillations in a Fermi liquid. ${ }^{1}$ From the experimental point of view, spectacular measurements via scanning tunneling microscopy (STM) of the local electronic environment on suitable metallic surfaces of threedimensional elemental metals ${ }^{2-6}$ and on artificially prepared two-dimensional quantum wells ${ }^{7}$ have allowed the visualization and quantification of phenomena that in the past have per se only raised purely academic or indirect interest. While current experimental work has focused on the local density of electronic states at the Fermi energy, it is quite reasonable to expect that deviations of the actual electronic number density from its equilibrium value will soon be amenable to direct measurement by means of the same powerful techniques. From the theoretical point of view the advent of elegant quantum diffusion Monte Carlo techniques has at the same time allowed the numerical exact determination of several response properties of the homogeneous electron liquid in both three and two dimensions. ${ }^{8-11}$ The connection of these two seemingly disparate fields of study will be discussed and exploited in this paper.

It is well known how, in the linear regime, the asymptotic behavior of the local electron density deviation $n_{1}(r)$ at a distance $r$ from a perturbing impurity reflects any nonanalytic behavior characterizing the Fourier transform $\chi(q)$ of the static density response function of an otherwise homogeneous electron liquid via the relation

$$
n_{1}(\mathbf{r})=\int e^{i \mathbf{q} \cdot \mathbf{r}} \chi(q) V_{e x t}(q) \frac{d^{d} q}{(2 \pi)^{d}}
$$

where $d=1,2,3$ and $V_{e x t}(q)$ is the Fourier transform of an isotropic impurity potential. This expression is in fact quite accurate for most values of $r$ except in the immediate vicinity of the impurity where $n_{1}$ becomes large enough to invalidate the linear approximation. Equation (1) can also be conveniently rewritten as
PACS number(s): 71.10.Ay, 71.10.Ca, 72.10.-d, 71.55.-i

$$
n_{1}(r)=\int_{0}^{\infty} \chi(q) V_{e x t}(q) F_{d}(q r) q^{d-1} d q
$$

where

$$
F_{d}(x)= \begin{cases}\frac{\cos (x)}{\pi}, & 1 \mathrm{D}, \\ \frac{J_{0}(x)}{2 \pi}, & 2 \mathrm{D}, \\ \frac{\sin (x)}{2 \pi^{2} x}, & 3 \mathrm{D},\end{cases}
$$

where in turn $J_{0}(x)$ is the zeroth-order Bessel function. ${ }^{12}$ Clearly as long as $V_{e x t}(q)$ is well behaved, the long-range behavior of $n_{1}(r)$ simply stems from general properties of Fourier transforms. ${ }^{13}$

For non-interacting electrons $\chi(q)$ coincides with the Lindhard function $\chi_{0}(q)$ (see also Secs. II A and II B). In this case, by making use of the explicit formulas for $\chi_{0}(q)$ (Ref. 14), it is a straightforward exercise to show that for $d=1,2,3$ the following general asymptotic formula applies for all well-behaved impurity potentials $V_{e x t}(q)$ :

$$
n_{1}(r) \approx \frac{\sin \left(2 k_{F} r+\phi_{d}\right)}{r^{d}},
$$

where $\phi_{d}$ is a suitable dimensionality-dependent phase. The characteristic power law decay $r^{-d}$ of this oscillatory part of the electron density distortion is a direct consequence of the well-known nonanalyticity of $\chi_{0}(q)$ for $q=2 k_{F}$, which in turns represents the signature of a metallic Fermi surface. A similar but more complicated phenomenon occurs for a twodimensional electron liquid in the presence of a Rashba type spin-orbit coupling. ${ }^{15}$ A study of this problem can be found in Refs. 16 and 17. As discussed in Sec. III, a striking counter-example to this behavior is represented by the case of two-dimensional electrons in a perpendicular quantizing magnetic field occupying a full Landau level for which the Lindhard function $\chi_{0}(q)$ is instead an analytic function of $q$. 
The way Coulomb interactions modify the non-interacting electrons result strongly depends on dimensionality. The reason is that while two- and three-dimensional interacting electrons have been observed to behave as Fermi liquids for a vast range of characterizing parameters (e.g., density), electrons in one space dimension behave like a Luttinger liquid. ${ }^{14,18,19}$

Consider first the case of two- and three-dimensional electronic Fermi liquids. In this case the problem at hand is rather simple and in fact amenable to an exact solution. Since by its own nature the low-lying excited states of a Fermi liquid are connected by continuity to those of the corresponding non-interacting system, it is quite reasonable to expect that no new nonanalytic behavior emerges in the wave vector dependence of the static response functions of the interacting system that is not already present in the noninteracting one. More formally one can arrive at the same conclusion by considering that, by Luttinger's theorem, the Fermi surface is a robust entity across the non-interacting to Fermi liquid isomorphism. It is then quite natural in the present context to make use of the exact relation: ${ }^{14}$

$$
\chi(q)=\frac{\chi_{0}(q)}{1-v_{q}\left[1-G_{+}(q)\right] \chi_{0}(q)},
$$

which expresses the fully interacting response function $\chi(q)$ in terms of the corresponding non-interacting Lindhard function $\chi_{0}(q)$ and the so-called static spin-symmetric manybody local field factor $G_{+}(q)$ in which all electron correlations beyond the standard random phase approximation [RPA, i.e., $\left.G_{+}(q)=0\right]$ are embedded. Here $v_{q}$ is the Fourier transform of the Coulomb interaction and is given by $v_{q}=2 \pi e^{2} / q$ in two dimensions and by $v_{q}=4 \pi e^{2} / q^{2}$ in three. This formula represents one of the fundamental relations within the general theory of the many-body local fields, ${ }^{14} \mathrm{a}$ scheme that has proved very successful in the theoretical determination of basic physical properties of experimentally accessible interacting electron liquids in both two $^{20}$ and three dimensions. $^{21}$

Clearly in the Fermi liquid state the only nonanalytic behavior of $\chi(q)$ must come from that of $\chi_{0}(q)$, the many-body local field factor, $G_{+}(q)$ being in this case a smooth analytic function of $q$. The latter has been recently quite accurately determined by Monte Carlo methods. ${ }^{8-11}$ Based on the results of these studies, interpolation formulas useful for the efficient use of the function $G_{+}(q)$ in various theoretical calculations have been developed both for three- ${ }^{22}$ as well as two-dimensional electronic systems ${ }^{23}$ for a wide range of electronic densities. Figure 1 displays the characteristic wave vector dependence of $G_{+}(q)$ in two- and three-dimensional electronic Fermi liquids in the homogeneous, paramagnetic state. The curves have been obtained by means of the formulas of Refs. 22 and 23. For illustration purposes we have chosen the same value $r_{s}=2$ for the electronic density parameter in both cases.

The expression of Eq. (5) will play a central role in our theory.

Although the reminder of this paper will be devoted to the study of two- and three-dimensional Fermi liquids it is use-

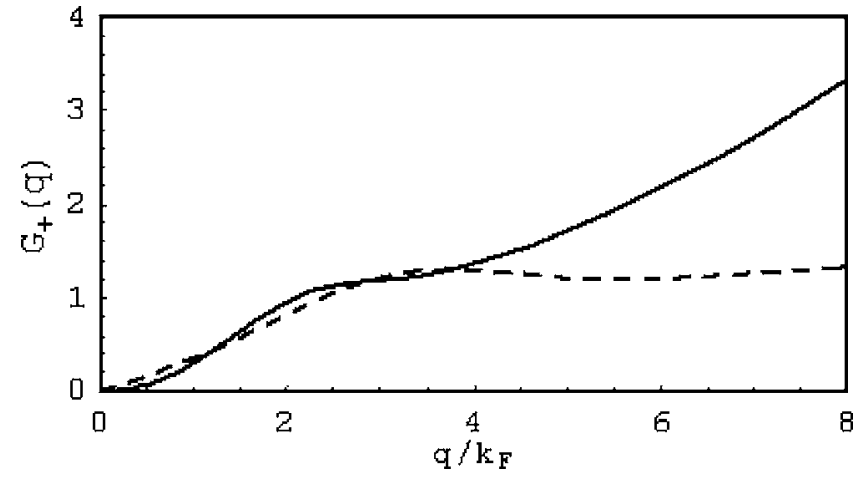

FIG. 1. The wave vector dependence of the static spinsymmetric many-body local field factor $G_{+}(q)$ for two- (dashed line) and three- (solid line) dimensional homogeneous, paramagnetic Fermi liquids as obtained from interpolation formulas of accurate quantum Monte Carlo data. Here $r_{s}=2$.

ful, as a comparison, to recall here what happens in the case of one-dimensional electronic systems in which the interactions lead to a truly nontrivial modification of the Friedel oscillation phenomenon. ${ }^{24}$ To begin with, we notice that, interestingly, in a Luttinger liquid, a behavior similar to that of Eq. (4) can be seen to still obtain for intermediate distances. A crucial difference is, however, that the power law decay is generally weaker. On the other hand, the actual long-range behavior of the perturbed electron density in this situation cannot be described within linear response theory. All direct calculations show that in this system, Friedel-type oscillations do still occur but their amplitude asymptotically decays with a power law characterized by an exponent that depends on the strength of the electron-electron interaction. ${ }^{14,25}$

The manuscript is organized as follows: in Sec. II we present the general results for the linear response theory of the density modulation $n_{1}(r)$ in two and three dimensions for the cases of (1) a coulombic impurity potential $V_{\text {ext }}(\vec{r})=Z e^{2} / r \quad$ for which $V_{\text {ext }}(q)=(d-1) 2 \pi Z e^{2} / q^{d-1}$, and (2) a local impurity potential $V_{e x t}(\vec{r})=C \delta(\vec{r})$ for which $V_{e x t}(q)=C$. In Sec. III we present and discuss the results of our analysis and draw our conclusions. Finally the counterexample of the case of two-dimensional electrons subject to a perpendicular quantizing magnetic field in discussed in the Appendix.

\section{GENERAL FORMULATION}

We begin by introducing a simplifying notation. We will express the Lindhard functions in both two and three dimensions as follows,

$$
\chi_{0}(q)=-N_{d}(0) u_{d}(x),
$$

where $x=q / k_{F}, d=2,3, u_{d}(x)$ is a function (whose explicit expressions will be given below), and $N_{d}(0)$ is the total density of states per unit volume at the Fermi energy for a noninteracting electron liquid in $d$ dimensions. We recall next the corresponding definition of the Thomas-Fermi screening wave vector: 


$$
\kappa_{d}^{d}=2 \pi(d-1) e^{2} N_{d}(0) .
$$

With this notation the expression of Eq. (5) for the fully interacting static charge response function can be written as

$$
\frac{\chi(q)}{N_{d}(0)}=-\frac{x^{d-1} u_{d}(x)}{x^{d-1}+\left(\frac{\kappa_{d}}{k_{F}}\right)^{d-1}\left[1-G_{+}\left(k_{F} x\right)\right] u_{d}(x)},
$$

where the factor $\left(\kappa_{d} / k_{F}\right)^{d-1}$ is seen to represent the dimensionless coupling constant of the problem.

In the same way the Fourier transform of the coulombic impurity potential can be expressed as

$$
V_{e x t}(q) N_{d}(0)=\frac{Z}{x^{d-1}}\left(\frac{\kappa_{d}}{k_{F}}\right)^{d-1} .
$$

These formulas will be used alongside Eq. (3) in the following sections to evaluate the quadrature of Eq. (2) in the cases of interest.

\section{A. Two-dimensional electron liquid}

In a two-dimensional electron liquid the Lindhard function $\chi_{0}(q)$ is obtained, via Eq. (6), in terms of $N_{2}(0)=m / \pi \hbar^{2}$ and the function ${ }^{14,26,29}$

$$
u_{2}(x)=1-\theta(x-2) \frac{\sqrt{x^{2}-4}}{x} .
$$

In this case, in terms of the Bohr radius and the density parameter $r_{s}, \kappa_{2}=2 / a_{B}$ and $k_{F}=\sqrt{2 \pi n}=\sqrt{2} / a_{B} r_{s}$ so that the coupling constant is given by $\kappa_{2} / k_{F}=\sqrt{2} r_{s}$.

We will consider two possible types of impurity potential: that of an isolated charged impurity with Coulomb potential $V_{e x t}(q)=2 \pi Z e^{2} / q$, and that of a localized impurity potential with $V_{e x t}(q)=C$. For both cases, by making use of Eqs. (2), (3), and (5), it is easily found that the linear response of the electronic number density is given by the integral

$$
\frac{n_{1}(r)}{n}=-A_{L(S) R} \int_{0}^{\infty} \frac{x^{N_{R}} u_{2}(x) J_{0}\left(k_{F} r x\right) d x}{x+\sqrt{2} r_{S}\left[1-G_{+}\left(k_{F} x\right)\right] u_{2}(x)},
$$

where both the power $N_{R}$ appearing in the numerator and the coefficient $A_{L(S) R}$ depend on what type of external potential is assumed (LR and SR stand for long and short range). In particular $N_{R}=1$ for the Coulomb impurity potential and $N_{R}=2$ for the localized potential. Moreover, the coefficient acquires for the two cases the following expressions:

$$
A_{L R}=\sqrt{2} Z r_{s}, \quad A_{S R}=\frac{C}{\pi e^{2} a_{B}} .
$$

\section{B. Three-dimensional electron liquid}

In a three-dimensional electron liquid the corresponding expressions are readily obtained in the same way. In this case $\chi_{0}(q)$ is determined by $N_{3}(0)=m k_{F} / m^{2} \hbar^{2}$, and ${ }^{14,27,29}$
TABLE I. The explicit expressions for the linear density modulation induced by a local impurity potential in a non-interacting three-, two-, and one-dimensional electron gas.

\begin{tabular}{cc}
\hline \hline & $\frac{n_{1}(r)}{n}$ \\
\hline $3 \mathrm{D}$ & $6 m k_{F} C / \hbar^{2} \pi \sin 2 k_{F} r-2 k_{F} r \cos 2 k_{F} r /\left(2 k_{F} r\right)^{4}$ \\
$2 \mathrm{D}$ & $m C / \hbar^{2}\left[J_{0}\left(k_{F} r\right) N_{0}\left(k_{F} r\right)+J_{1}\left(k_{F} r\right) N_{1}\left(k_{F} r\right)\right]$ \\
$1 \mathrm{D}$ & $m C / \hbar^{2} k_{F} s i\left(2 k_{F} x\right)$ \\
\hline \hline
\end{tabular}

$$
u_{3}(x)=\frac{1}{2}+\frac{x^{2}-4}{8 x} \ln \left|\frac{x-2}{x+2}\right|
$$

Moreover, $\quad \kappa_{3}^{2}=4 \pi e^{2} N_{3}(0)=\left(4 \pi a_{B} r_{s}\right)(9 \pi / 4)^{1 / 3}$, $k_{F}=\left(3 \pi^{2} n\right)^{1 / 3}=(9 \pi / 4)^{1 / 3}\left(1 / a_{B} r_{S}\right)$, and therefore the coupling constant is given by $\kappa_{3}^{2} / k_{F}^{2}=(4 / \pi)(4 / 9 \pi)^{1 / 3} r_{s}$.

Here the linear density modulation is determined by the following quadrature:

$$
\frac{n_{1}(r)}{n}=\frac{B_{L(S) R}}{k_{F} r} \int_{0}^{\infty} \frac{x^{N_{R}} u_{3}(x) \sin \left(k_{F} r x\right) d x}{x^{2}+\frac{4}{\pi}\left(\frac{4}{9 \pi}\right)^{1 / 3} r_{s}\left[1-G_{+}\left(k_{F} x\right)\right] u_{3}(x)} .
$$

In this case $N_{R}=1$ for the Coulomb impurity potential and $N_{R}=3$ for the case of the localized potential. The coefficients acquire here the following expressions:

$$
B_{L R}=-\frac{6}{\pi}\left(\frac{4}{9 \pi}\right)^{1 / 3} Z r_{S}, \quad B_{S R}=-\frac{16(4 \pi)^{1 / 3} C}{9 e^{2} r_{s} a_{B}^{2}},
$$

respectively, for the Coulomb (LR) and delta function (SR) interaction.

The results of the numerical study as well as the derivation of the closed form formulas for the corresponding asymptotic behavior of $n_{1}(r)$ in two and three dimensions are reported and discussed next in Sec. III.

\section{RESULTS, DISCUSSION, AND CONCLUSIONS}

The general formulas determining the linear response of the electronic density to a perturbing impurity potential having been derived, we proceed next to a discussion of the results.

\section{A. Non-interacting electrons}

The limit of non-interacting electrons can be immediately obtained by setting the coupling constant to zero in Eqs. (11) and (14). For the particular case of a local (delta function) impurity potential, the calculations amount to Fourier transforming the Lindhard function and can be carried out analytically. The results are known and, for completeness sake, we report them in Table I where we have also included for reference the one-dimensional formula. ${ }^{14,24,29}$ To arrive at this particular result avoiding some mathematical pitfalls ${ }^{24}$ it 


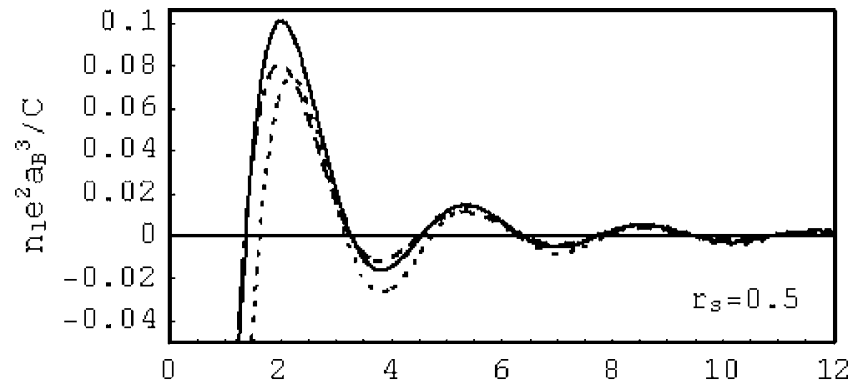

(a)
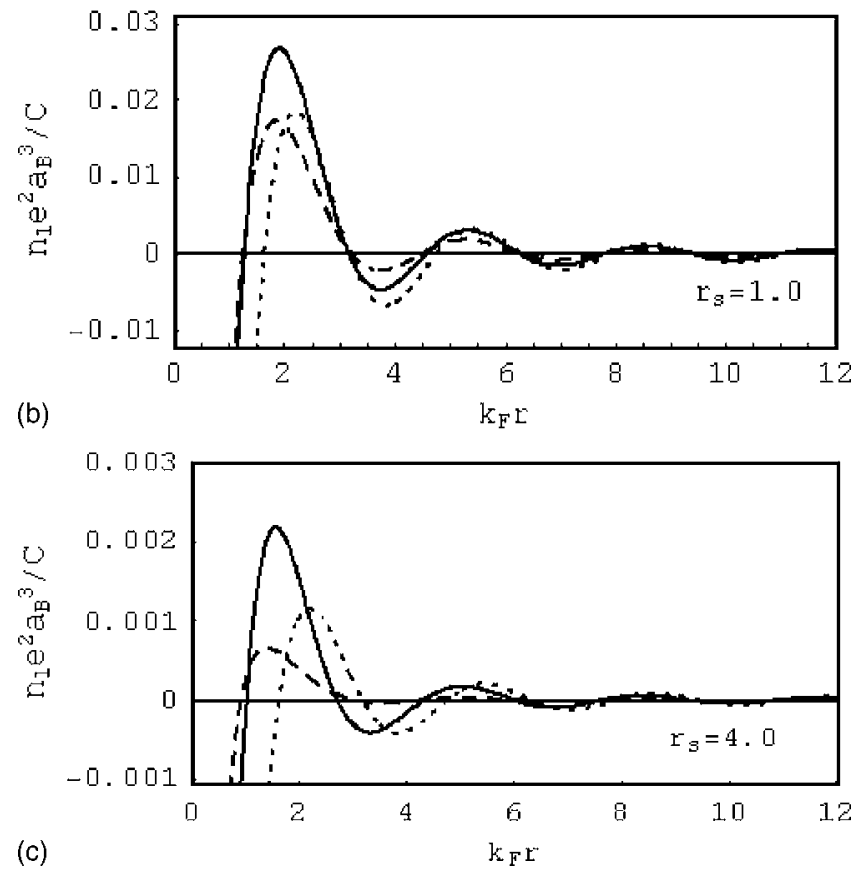

FIG. 2. The linear density modulation due to a short-range impurity potential in a two-dimensional electron gas for various densities. Solid lines: fully interacting; dashed lines: RPA; dotted lines: non interacting. Top panel: $r_{s}=0.5$. Middle panel: $r_{s}=1.0$. Bottom panel: $r_{s}=4.0$.

is best to simply Fourier transform the Lindhard function, which in this case is given by combining

$$
u_{1}(x)=\frac{1}{x} \ln \left|\frac{x+2}{x-2}\right|,
$$

with $N_{1}(0)=2 m / \pi \hbar^{2} k_{F}$. In Table I the Bessel functions and the sine integral function are defined as in Ref. 12. It is a simple exercise to show that all the formulas of Table I lead to the general behavior of Eq. (4).

Where appropriate, for the sake of comparison, plots corresponding to these formulas have been included as dotted lines in the figures below.

\section{B. General results for the density modulation}

Allowing for the electron-electron interaction within the random phase approximation simply amounts to the assumption $G_{+}\left(k_{F} x\right)=0$ in all our formulas. In the general fully interacting case, on the other hand, the integrals of Eqs. (11)
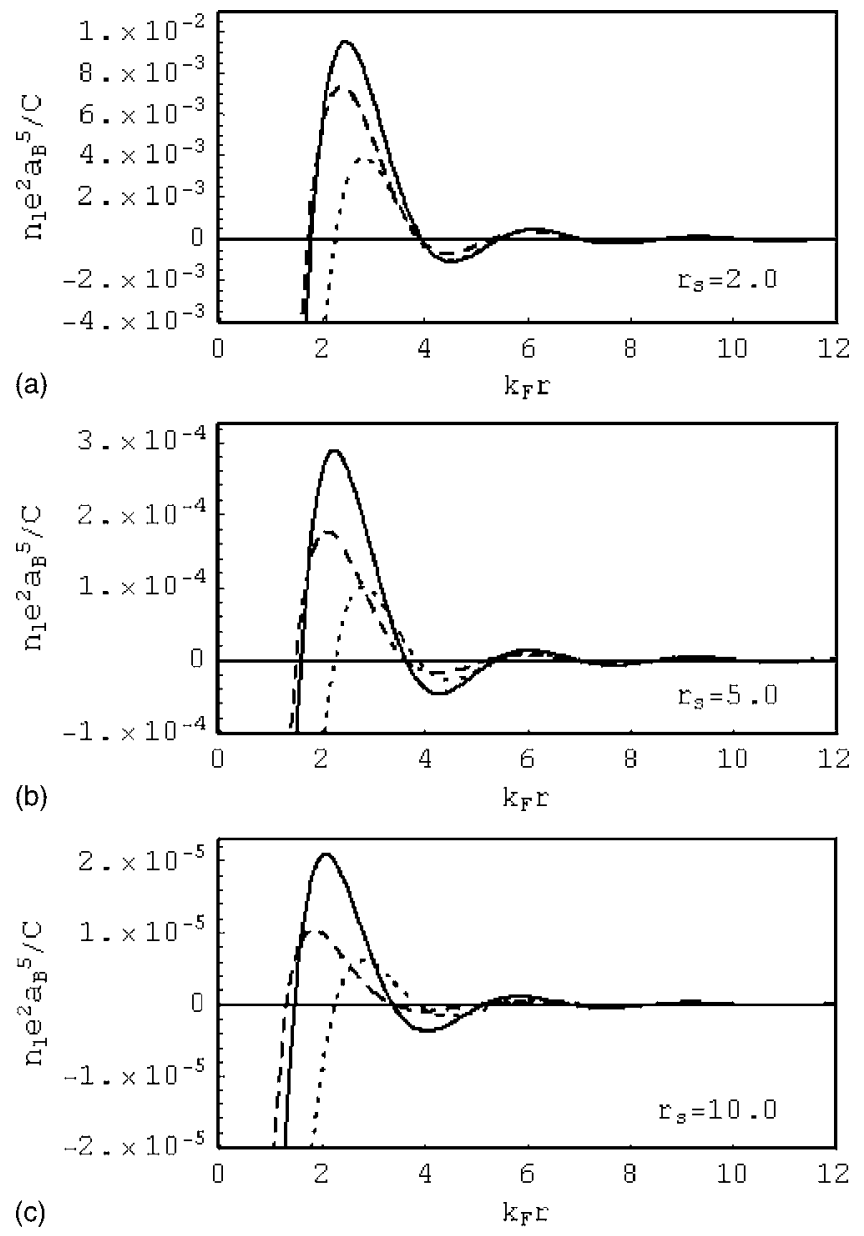

FIG. 3. The linear density modulation due to a short range impurity potential in a three-dimensional electron gas for various densities. Solid lines: fully interacting; dashed lines: RPA. Top panel: $r_{s}=2.0$. Middle panel: $r_{s}=5.0$. Bottom panel: $r_{s}=10.0$.

and (14) can be performed numerically once an appropriate analytic form for the many-body local field $G_{+}\left(k_{F} x\right)$ is employed. In our calculations we have made use of the explicit interpolation formulas proposed in Refs. 23 and 22, respectively, for the two- and three-dimensional cases.

The numerical integrations are straightforward. The results for the case of a local impurity potential for various values of the density parameter $r_{s}$ are displayed in Fig. 2 for the two-dimensional case and in Fig. 3 for the threedimensional one. In a similar way Figs. 4 and 5 display instead our results for the corresponding case of a long-range (Coulomb) impurity potential.

In Figs. 2 and 4 we plot the fully interacting case as a solid line, while the approximate RPA result is represented by a dashed line. Moreover, in Fig. 2 the non-interacting electrons result is provided (dotted line) for the sake of comparison.

\section{Asymptotic formulas}

Closed form expressions can be obtained for the asymptotic form of $n_{1}(r)$ for large values of $k_{F} r$. This can be readily reduced to the study of the asymptotic behavior of a 

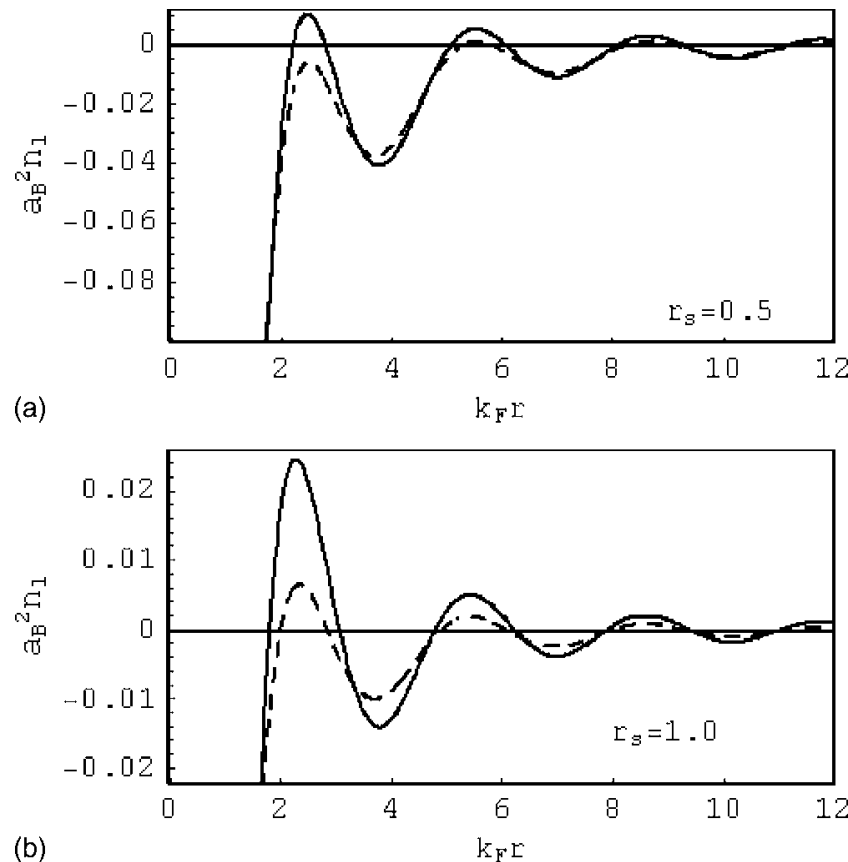

(b)

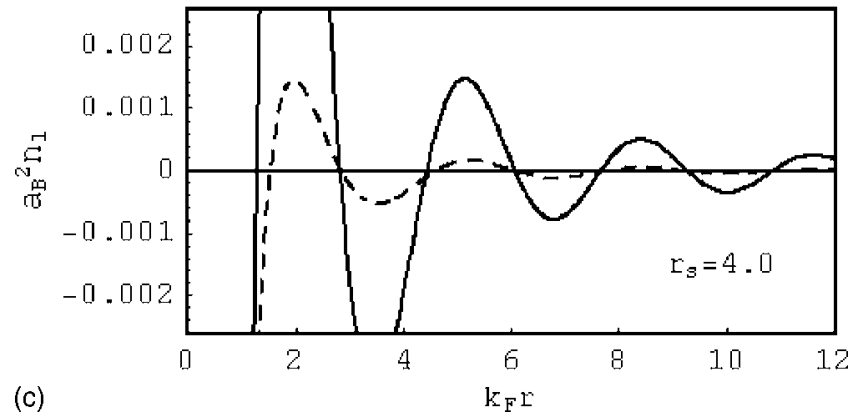

FIG. 4. The linear density modulation due to a long-range impurity potential in a two-dimensional electron gas for various densities $(Z=1)$. Top panel: $r_{s}=0.5$. Middle panel: $r_{s}=1.0$. Bottom panel: $r_{s}=4.0$.

Fourier transform. In the two-dimensional case this is accomplished by using in Eq. (11) the asymptotic expression for the zeroth-order Bessel function $J_{0}(y)$, i.e.,

$$
J_{0}(y) \simeq \sqrt{\frac{2}{\pi y}} \cos \left(y-\frac{\pi}{4}\right), \quad y \gg 1 .
$$

The sought results can then be either inferred by careful direct inspection or by making use of Lighthill theorem. ${ }^{13}$ Table II provides a straightforward application of the theorem to the cases relevant to this study. There $\widetilde{f}(y)$ is the standard Fourier transform of $f(x)$.

The results can be compactly summarized by the following formulas:

$$
\frac{n_{1}(r)}{n} \simeq \frac{\delta_{d L(S) R}}{\left[1+2\left(\frac{\kappa_{d}}{4 k_{F d}}\right)^{d-1}\left[1-G_{+}\left(2 k_{F}\right)\right]\right]^{2}} \frac{\sin \left(2 k_{F} r+\phi_{d}\right)}{r^{d}}
$$

where
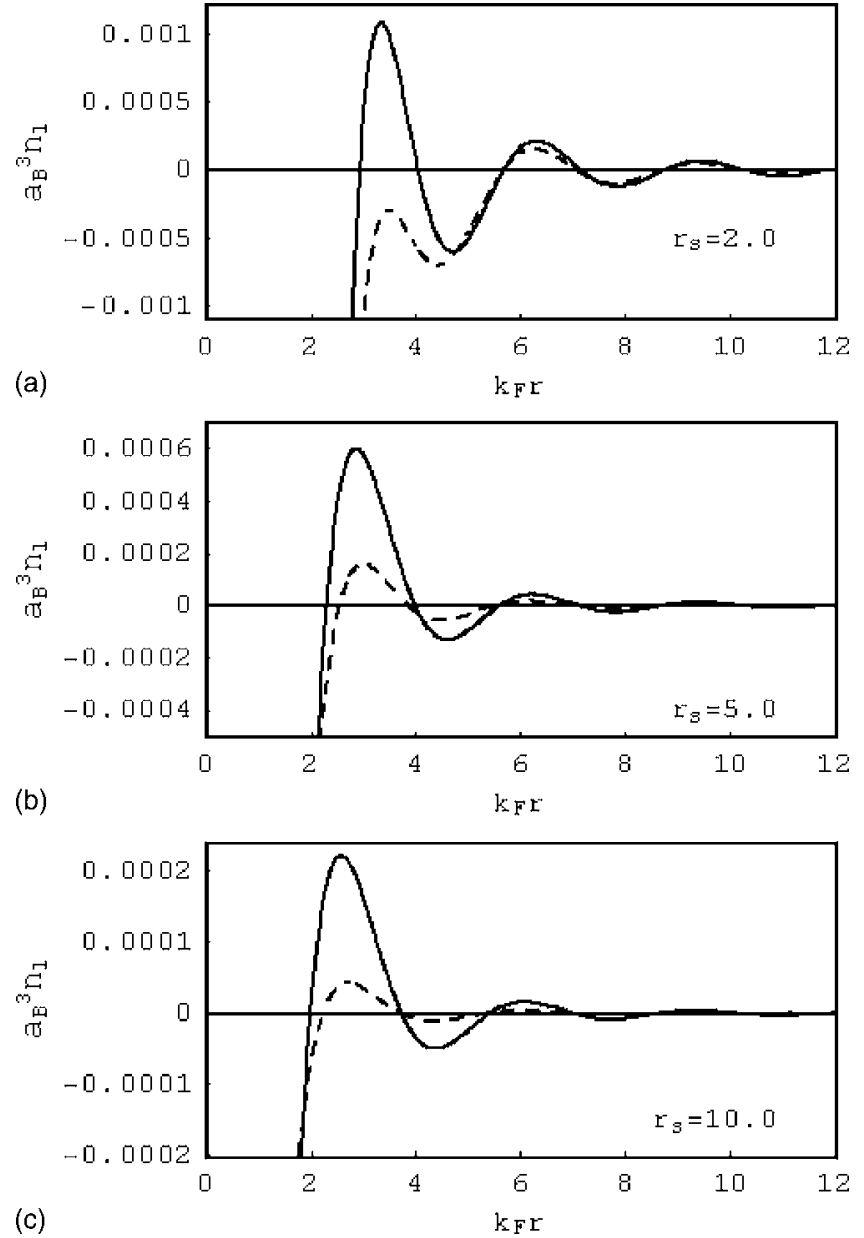

FIG. 5. The linear density modulation due to a long-range impurity potential in a three-dimensional electron gas for various densities $(Z=1)$. Solid lines: fully interacting; dashed lines: RPA. Top panel: $r_{s}=2.0$. Middle panel: $r_{s}=5.0$. Bottom panel: $r_{s}=10.0$.

$$
\begin{array}{cc}
\delta_{3 S R}=\frac{32(4 \pi)^{1 / 3} a_{B} r_{s}^{2} C}{81 \pi e^{2}}, & \delta_{2 S R}=\frac{a_{B} r_{s}^{2} C}{2 \pi e^{2}}, \\
\delta_{3 L R}=\frac{a_{B}^{3} r_{s}^{4} Z}{3 \pi}\left(\frac{4}{9 \pi}\right)^{1 / 3}, & \delta_{2 L R}=\frac{a_{B}^{2} r_{s}^{3} Z}{2 \sqrt{2}},
\end{array}
$$

and

$$
\phi_{2}=\pi, \quad \phi_{3}=\frac{3}{2} \pi
$$

TABLE II. The applications of the Lighthill theorem for the determination of the asymptotic behavior of the linear density modulation in one, two, and three dimensions.

\begin{tabular}{cc}
\hline \hline$f(x)$ & Asymptotic behavior of $\tilde{f}(y)$ \\
\hline $\ln |x-2|$ & $-\pi /|y| e^{2 i y}$ \\
$\theta(x-2) \sqrt{|x-2|}$ & $-\sqrt{\pi} / 2|y|^{3 / 2} e^{i(2 y+\pi / 4)}$ \\
$(x-2) \ln |x-2|$ & $-i \pi \operatorname{sgn}(y) / y^{2} e^{2 i y}$ \\
\hline \hline
\end{tabular}



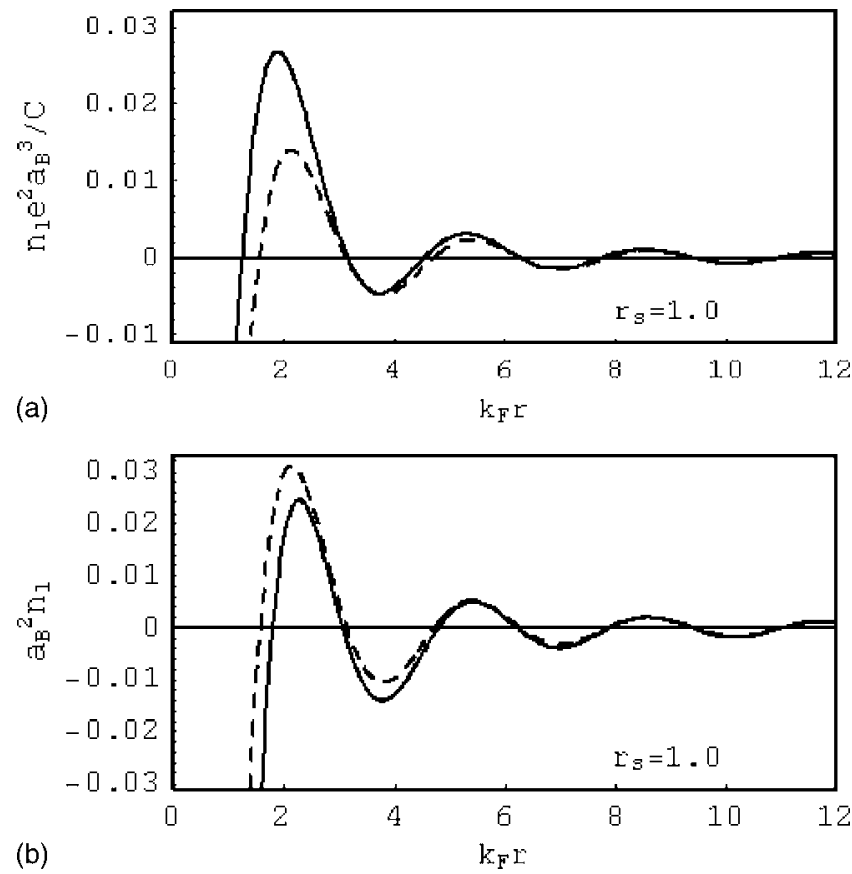

FIG. 6. The comparison between the leading term (dashed line) and the exact expression (solid line) of the linear density modulation due to a short-range impurity potential in a fully interacting two- dimensional electron gas with $r_{s}=1.0$. Top panel: short range impurity potential. Bottom panel: long range impurity potential.

Of course, one can calculate corrections to this leading order term. They are of order $r^{-5 / 2}$ for $2 \mathrm{D}$ electron gas and $r^{-4}$ for the 3D electron gas.

\section{Discussion and conclusions}

We have determined the exact value of the linear density response of an electron liquid in two and three dimensions to the potential of an isolated impurity. It is clear that our results have physical meaning for distances sufficiently large as to validate the linear response approximation. To quantify this requirement we note that for a two-dimensional electron liquid, the ratio $n_{1} / n$ at the first peak of the density modulation for $r_{s}=1$, equals $0.07 Z$ for the long-range impurity potential and $0.17 C /\left(e^{2} a_{B}\right)$ for the delta function potential (Figs. 4 and 2, middle panel). In three dimensions for $r_{s}=2$ the corresponding $n_{1} / n$ ratios for the first visible peaks in Figs. 5 and 3 (top panel) respectively acquire the values $0.013 Z$ and $0.3 C /\left(e^{2} a_{B}^{2}\right)$.

An inspection of Figs. 2 and 4 shows that many-body effects beyond the simple RPA are very important for the accurate determination of the amplitude and position of the first density crest and valley, a fact that, as one would expect, proves increasingly true for decreasing electron densities. ${ }^{28}$ This is crucial when comparing theory with experiment where, in most cases, only the very first oscillations about an impurity can be quantitatively experimentally characterized. ${ }^{7}$ Conversely an accurate characterization of the amplitude of the oscillations can be used to determine the value of the quantity $G_{+}\left(k_{F} x\right)$. This would not only allow us to test the validity and accuracy of the Quantum Monte Carlo based

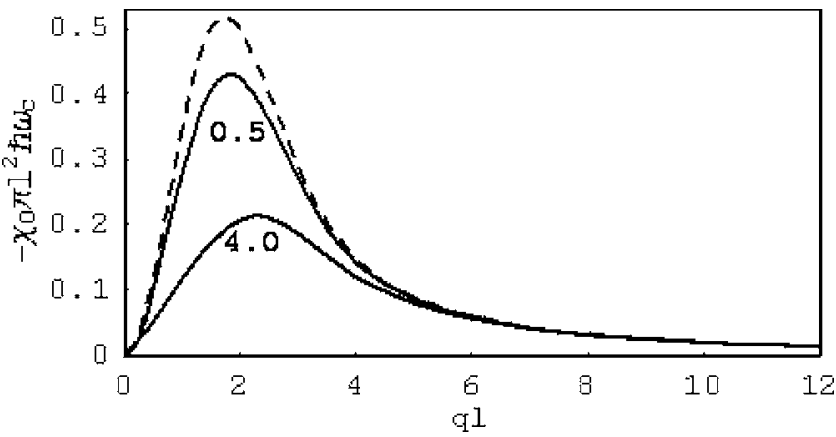

FIG. 7. The density response function in a quantizing magnetic field. Solid lines: RPA (upper curve: $\ell / a_{B}=0.5$, lower curve $\left.\ell / a_{B}=4.0\right)$. Dotted line: free electrons.

calculations of the electron liquid, but also provide an indication of the proximity of the system to a compressibility related instability. ${ }^{14}$

It is very useful to establish the accuracy of the asymptotic formula of Eq. (18), i.e., one should try to determine how quickly the density modulation conforms to its asymptotic behavior. What we find is that the latter is quickly established after just one or two oscillations. For the particular case of the two-dimensional electronic system, this is shown in Fig. 6 for both short- and long-range impurity potentials. It should be noted that this early conformity is eventually spoiled at low electron densities.

As already mentioned, for an electronic system to exhibit Friedel oscillations characterized by the power law decay of Eq. (4), the Fermi surface must exist and be of metallic type with a finite density of states at the Fermi energy. Accordingly the observation of Friedel oscillations of this type can

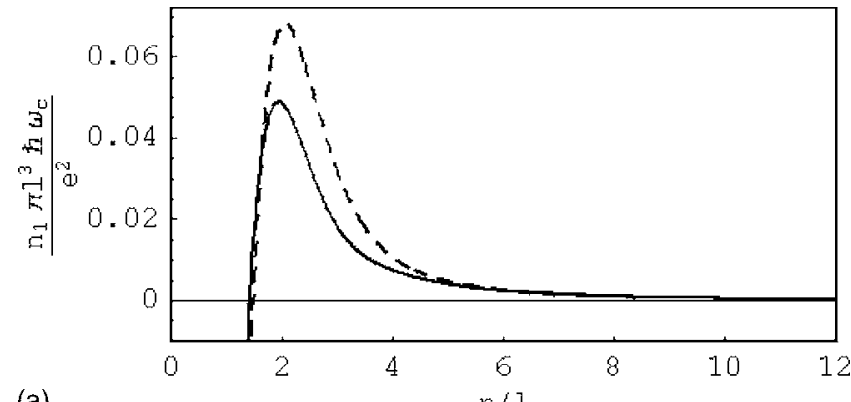

(a)

$r / 1$

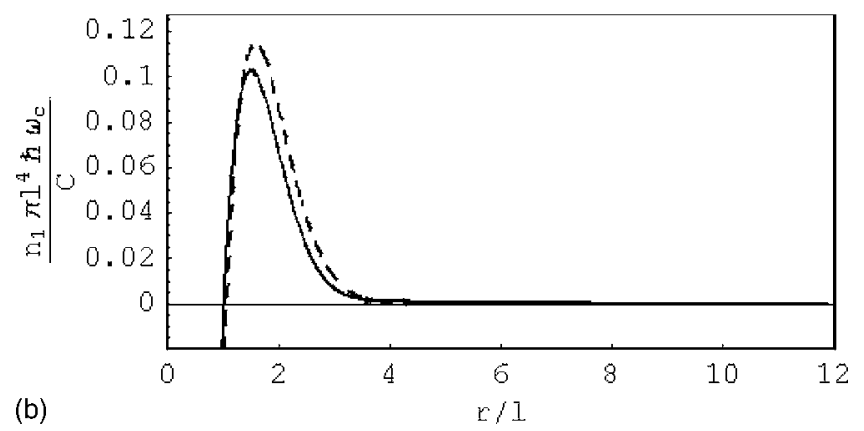

FIG. 8. The linear density modulation due to an isolated impurity in a two-dimensional electron liquid for $\ell / a_{B}=0.5$. Solid line: RPA; dashed line: free electrons. Top panel: short-range impurity potential. Bottom panel: long-range impurity potential. 
be seen as a signature of the Fermi liquid state.

It is quite interesting and illuminating to compare this situation to that of the density response to an isolated static impurity of a two-dimensional electron liquid in the presence of a strong quantizing transverse magnetic field in the limit in which only the lowest Landau level is occupied. In this case the electronic system is characterized by a vanishing density of states at the Fermi surface, the corresponding static Lindhard function is an analytic function of $q$, and there are no Friedel oscillations. This problem can be solved with very high accuracy within the RPA and the details are provided in the Appendix.

\section{ACKNOWLEDGMENTS}

The authors would like to acknowledge useful discussions with A. W. Overhauser. GS work was partially supported by a grant from the Purdue Research Foundation.

\section{APPENDIX: TWO-DIMENSIONAL ELECTRONS IN QUANTIZING MAGNETIC FIELD}

Consider a non-interacting two-dimensional electron liquid subject to a quantizing perpendicular static magnetic. Assuming that the electrons fully occupy a certain set of Landau levels, and that there is no sizable Zeeman coupling, the corresponding static Lindhard function is given by

$$
\chi_{0}(q)=\frac{1}{2 \pi \ell^{2}} \sum_{\sigma, n, n^{\prime}} \frac{f\left(\varepsilon_{n}\right)-f\left(\varepsilon_{n^{\prime}}\right)}{\left(n-n^{\prime}\right) \hbar \omega_{c}}\left|F_{n^{\prime}, n}(q)\right|^{2},
$$

where $\omega_{c}$ is the cyclotron frequency, $\ell=\sqrt{\hbar c / e B}$ is the magnetic length and we have defined

$$
F_{n^{\prime}, n}(\mathbf{q})=\sqrt{\frac{n !}{n^{\prime} !}}\left(\frac{\left(q_{y}-i q_{x}\right) \ell}{\sqrt{2}}\right)^{n^{\prime}-n} e^{-q^{2} \ell^{2} / 4} L_{n}^{n^{\prime}-n}\left(\frac{q^{2} \ell^{2}}{2}\right)
$$

with $L_{n}^{n^{\prime}-n}(x)$ a generalized Laguerre polynomial. ${ }^{12}$ If only the lowest Landau level is occupied, then

$$
\chi_{0}(q)=-\sum_{n>0} \frac{\left|F_{n, 0}(\mathbf{q})\right|^{2}}{\pi \ell^{2} n \hbar \omega_{c}}=-\frac{e^{-q^{2} \ell^{2} / 2}}{\pi \ell^{2} \hbar \omega_{c n>0}} \sum_{n>0} \frac{\left(q^{2} \ell^{2}\right)^{n}}{2^{n} n ! n} .
$$

The last sum can be readily performed giving

$$
\chi_{0}(q)=-\frac{e^{-q^{2} \ell^{2} / 2}}{\pi \ell^{2} \hbar \omega_{c}}\left[E i\left(\frac{q^{2} \ell^{2}}{2}\right)-\ln \left(\frac{q^{2} \ell^{2}}{2}\right)-\gamma\right],
$$

where the exponential integral function $\operatorname{Ei}(x)$ is defined as

$$
\operatorname{Ei}(x)=-\mathcal{P} \mathcal{V} \int_{-x}^{\infty} \frac{e^{-t}}{t} d t,
$$

and $\gamma$ is the Euler-Mascheroni constant. ${ }^{12}$

This function is plotted as a dashed line in Fig. 7. Notice that in this case $\chi_{0}(q)$ is an analytic function of $q$ with limiting behaviors given by

$$
\chi_{0}(q) \simeq\left\{\begin{array}{l}
-\frac{q^{2}}{2 \pi \hbar \omega_{c}}, \quad q \rightarrow 0, \\
-\frac{2}{\pi \ell^{4} q^{2} \hbar \omega_{c}}, \quad q \rightarrow \infty .
\end{array}\right.
$$

Because $\chi_{0}(q)$ is analytic there are no Friedel oscillations. The density response is evaluated using Eq. (2) for both short- and long-range impurity potential:

$$
\begin{aligned}
n_{1}= & -\frac{\eta_{L(S) R}}{\pi \ell^{3} \hbar \omega_{c}} \int_{0}^{\infty}\left[E i\left(x^{2}\right)-\ln \left(x^{2}\right)\right. \\
& -\gamma] e^{-x^{2}} J_{0}\left(x \frac{r \sqrt{2}}{\ell}\right) x^{N_{L(S) R}} d x,
\end{aligned}
$$

where $a=\sqrt{2} e^{2} / \ell \hbar \omega_{c}=\sqrt{2} \ell / a_{B}, N_{S R}=0, N_{L R}=1, \eta_{L R}=Z \sqrt{2} e^{2}$, and $\eta_{S R}=C / \pi \ell$.

In the case of a Coulomb potential the density modulation decreases as $1 / r^{3}$ at large distances. For a localized potential, the result of the integration can be expressed in terms of special functions as follows:

$$
\begin{aligned}
n_{1}(r)= & \frac{C}{\pi^{2} \ell^{4} \hbar \omega_{c}}\left\{\left.\frac{4 \ell^{2} \pi}{r^{2}} G_{2,3}^{2,1}\left(\frac{r^{2}}{2 \ell^{2}}\right)\right|_{1,1,1 / 2} ^{1,1 / 2}\right. \\
& \left.-\left[E i\left(\frac{r^{2}}{2 \ell^{2}}\right)-\ln \left(\frac{r^{2}}{2 \ell^{2}}\right)-\gamma\right] e^{-r^{2} / 2 \ell^{2}}\right\},
\end{aligned}
$$

where $G_{2,3}^{2,1}$ is a Meijer function. ${ }^{12}$ The density modulation behaves as $2 / \pi^{2} r^{4} \hbar \omega_{c}$ for large distances.

The density response can be evaluated in RPA following the standard procedure. ${ }^{14}$ The result for both long- and shortrange impurity potentials can be compactly expressed in terms of the following quadrature:

$$
\begin{aligned}
n_{1}(r)= & -\frac{\eta_{L(S) R}}{\pi \ell^{3} \hbar \omega_{c}} \int_{0}^{\infty} \frac{\left[E i\left(x^{2}\right)-\ln \left(x^{2}\right)-\gamma\right] e^{-x^{2}}}{1+\frac{a}{x}\left[\operatorname{Ei}\left(x^{2}\right)-\ln \left(x^{2}\right)-\gamma\right] e^{-x^{2}}} \\
& \times J_{0}\left(x \frac{r \sqrt{2}}{\ell}\right) x^{N_{L(S) R}} d x
\end{aligned}
$$

where the notation was defined in Eq. (28). For illustration purposes the result of the numerical evaluation of this formula is plotted in Fig. 8 . 
${ }^{1}$ J. Friedel, Adv. Phys. 3, 446 (1954).

${ }^{2}$ M. Crommie, C. Lutz, and D. Eigler, Nature (London) 363, 524 (1993).

${ }^{3}$ G. A. Fiete, Phys. Rev. Lett. 86, 2392 (2001).

${ }^{4}$ B. G. Briner, P. Hofmann, M. Doering, H.-P. Rust, E. W. Plummer, and A. M. Bradshaw, Phys. Rev. B 58, 13931 (1998).

${ }^{5}$ L. Petersen, P. T. Sprunger, P. Hofmann, E. Laegsgaard, B. G. Briner, M. Doering, H. P. Rust, A. M. Bradshaw, F. Besenbácher, and E. W. Plummer, Phys. Rev. B 57, R6858 (1998).

${ }^{6}$ P. Hofmann, B. G. Briner, M. Doering, H.-P. Rust, E. W. Plummer, and A. M. Bradshaw, Phys. Rev. Lett. 79, 265 (1997).

${ }^{7}$ K. Kanisawa, M. J. Butcher, H. Yamaguchi, and Y. Hirayama, Phys. Rev. Lett. 79, 265 (1997).

${ }^{8}$ S. Moroni, D. M. Ceperley, and G. Senatore, Phys. Rev. Lett. 69, 1837 (1992).

${ }^{9}$ S. Moroni, D. M. Ceperley, and G. Senatore, Phys. Rev. Lett. 75, 689 (1995).

${ }^{10}$ G. Ortiz, M. Harris, and P. Ballone, Phys. Rev. Lett. 82, 5317 (1999).

${ }^{11}$ G. Senatore, S. Moroni, and D. Ceperley, in Quantum Monte Carlo Methods in Physics and Chemistry, edited by M. P. Nightingale and C. J. Umrigar (Kluwer, Dordrecht, 1999).

${ }^{12}$ I. Gradshteyn and I. Ryzhik, Table of Integrals, Series and Products (Academic Press, San Francisco, 1965).

${ }^{13}$ See for instance Table II in the text, and M. J. Lighthill, Introduction to Fourier Analysis and Generalised Functions (Cambridge University Press, Cambridge, 1958).
${ }^{14}$ G. F. Giuliani and G. Vignale, Quantum Theory of the Electron Liquid (Cambridge University Press, Cambridge, 2005).

${ }^{15}$ E. Rashba, Sov. Phys. Solid State 2, 1109 (1960).

${ }^{16}$ G.-H. Chen and M. E. Raikh, Phys. Rev. B 59, 5090 (1999).

${ }^{17}$ G. E. Simion and G. F. Giuliani, (unpublished).

${ }^{18}$ F. D. M. Haldane, J. Phys. C 14, 2585 (1981).

${ }^{19}$ A. Luther and I. Peschel, Phys. Rev. B 9, 2911 (1974).

${ }^{20}$ R. Asgari, B. Davoudi, M. Polini, G. F. Giuliani, M. P. Tosi, and G. Vignale, Phys. Rev. B 71, 045323 (2005).

${ }^{21}$ G. E. Simion and G. F. Giuliani, (unpublished).

${ }^{22}$ M. Corradini, R. Del Sole, G. Onida, and M. Palummo, Phys. Rev. B 57, 14569 (1998).

${ }^{23}$ B. Davoudi, M. Polini, G. F. Giuliani, and M. P. Tosi, Phys. Rev. B 64, 153101 (2001).

${ }^{24}$ Surprisingly the problem of the Friedel oscillations in a onedimensional Fermi liquid presents an interesting quirk of its own. See, for instance, G. F. Giuliani, G. Vignale, and T. Datta, (unpublished).

${ }^{25}$ R. Egger and H. Grabert, Phys. Rev. Lett. 75, 3505 (1995).

${ }^{26}$ F. Stern, Phys. Rev. Lett. 18, 546 (1967).

${ }^{27}$ J. S. Langer and S. H. Vosko, J. Phys. Chem. Solids 12, 196 (1959).

${ }^{28}$ For much larger values of $r_{s}$ the interactions are so strong that one expects the Fermi liquid picture to collapse, the electronic system eventually forming a Wigner solid.

${ }^{29}$ D. N. Aristov, Phys. Rev. B 55, 8064 (1997). 